\author{
$\underset{\text { SOCIÉTÉ }}{\text { CANADIAN }} \neq \begin{aligned} & \text { THORACIC SOCIETY } \\ & \text { CANADIENNE DE THORACOLOGIE }\end{aligned}$
}

\section{ANNOUNCEMENTS AND EVENTS}

\section{Announcements}

The Canadian Thoracic Society (CTS) held a very successful and well-attended Annual Scientific Program meeting at CHEST 2006, in Salt Lake City, on October 23 and 24. A number of lectures were held, most notably the Annual Christie Memorial Lecture, delivered by Dr Peter Paré, and the inaugural Institute of Circulatory and Respiratory Health Distinguished Lecture in Respiratory Sciences, delivered by Dr François Maltais. The CTS extends its gratitude to both of these lecturers as well as to Drs William Geerts, John Fleetham, Doug Helmersen, Andrew McIvor, Sanjay Mehta and Elizabeth Tullis who also delivered lectures as part of the 2006 scientific program meeting.

The Society also gratefully acknowledges the support of the following sponsors who helped ensure the success of the 2006 CTS Annual Scientific Program meeting:

\section{GOLD LeVEL SPONSORS: \\ AstraZeneca Canada Inc \\ GlaxoSmithKline Inc \\ Altana Pharma Inc}

Silver LeVEl SPONSORS:

Novartis Pharmaceuticals Canada Inc

Bayer HealthCare

Boehringer Ingelheim (Canada) Ltd / Pfizer Canada

BronZe LeVEl SpONSORS:

Actelion Pharmaceuticals Ltd

The Lung Association presents the 27th annual

Better Breathing Conference -

Lung Health: The Evolution of Lung Health February 2-3, 2007

Toronto Marriott Downtown Eaton Centre

525 Bay Street, Toronto, Ontario M5G 2L2

For more information, please contact the Ontario Thoracic

Society office, telephone 416-864-9911 ext 254 or e-mail ots@on.lung.ca.

\section{CALENDAR OF EVENTS - 2007}

January 21-24, Maui, Hawaii

Hawaii Thoracic Society's 7th Annual Symposium: Current Concepts in Pulmonary and Critical Care Maui, Hawaii

Web site http://ala-hawaii.org/2007-symposium.asp

\section{ANNONCES ET ÉVÉNEMENTS}

\section{Annonces}

La Société canadienne de thoracologie (SCT) a organisé un programme scientifique annuel fort réussi et fort prisé au congrès CHEST 2006 à Salt Lake City, les 23 et 24 octobre. Plusieurs conférences ont été présentées, notamment la conférence commémorative annuelle Christie, prononcée par le docteur Peter Paré, et la conférence inaugurale éminente de l'Institut de la santé respiratoire et circulatoire en sciences respiratoires, prononcée par le docteur François Maltais. La SCT remercie ces deux conférenciers, de même que les docteurs William Geerts, John Fleetham, Doug Helmersen, Andrew McIvor, Sanjay Mehta et Elizabeth Tullis, qui ont également donné des conférences dans le cadre du programme scientifique 2006.

La Société remercie également les commanditaires suivants, qui ont contribué à la réussite $\mathrm{du}$ programme scientifique annuel 2006 de la SCT :

\section{COMMANDITAIRES OR AstraZeneca Canada Inc. GlaxoSmithKline Inc. Altana Pharma Inc.}

Commanditaires Argent

Novartis Pharma Canada Inc.

Bayer HealthCare

Boehringer Ingelheim (Canada) Ltd. / Pfizer Canada

COMMANDiTAIRES BRONZE

Actelion Pharmaceuticals Ltd.

\author{
Better Breathing Conference - \\ Lung Health : The Evolution of Lung Health \\ Les 2 et 3 février 2007 \\ Toronto Marriott Downtown Eaton Centre \\ 525 Bay Street, Toronto (Ontario) M5G 2L2 \\ Renseignements : Bureau de la Société thoracique \\ de l'Ontario, \\ Téléphone : 416-864-9911, poste 254 \\ Courriel : ots@on.lung.ca
}

\section{CALENDRIER DES ÉVÉNEMENTS - 2007 \\ Du 21 au 24 janvier, Maui (Hawaï) \\ $7^{\mathrm{e}}$ symposium annuel de l'Hawaii Thoracic Society \\ Concepts actuels en soins pulmonaires et intensifs Maui (Hawaï) \\ Site Web : www.//ala-hawaii.org/2007-symposium.asp}


Better Breathing Conference -

Lung Health: The Evolution of Lung Health

February 2-3, 2007

Toronto Marriott Downtown Eaton Centre

525 Bay Street, Toronto, Ontario M5G 2L2

For more information, please contact the Ontario Thoracic

Society office, telephone 416-864-9911 ext 254 or e-mail ots@on.lung.ca.

European Respiratory Society Annual Congress

September 15-19, Stockholm, Sweden

Web: www.ersnet.org/ers/default.aspx?id=3924

\section{ON-LINE JOURNAL ACCESS}

For instructions on accessing the Canadian Respiratory Journal on-line, please see page 432 .

\section{WE'VE MOVED!}

Our new address is:

Canadian Thoracic Society

1750 Courtwood Crescent, Suite 300

Ottawa, Ontario

K2C 2B5

MOVING? PLEASE LET US KNOW - CONTACT:

Canadian Thoracic Society

1750 Courtwood Crescent, Suite 300

Ottawa, Ontario

K2C 2B5

Telephone: 613-569-6411 ext 229

Fax: 613-569-8860

E-mail: sdesmarais@lung.ca

\section{Better Breathing Conference}

Lung Health : The Evolution of Lung Health

Les 2 et 3 février 2007

Toronto Marriott Downtown Eaton Centre

525 Bay Street, Toronto (Ontario) M5G 2L2

Renseignements : Bureau de la Société thoracique de l'Ontario,

Téléphone : 416-864-9911, poste 254

Courriel : ots@on.lung.ca

Congrès annuel de la European Respiratory Society

du 15 au 19 septembre, Stockholm (Suède)

Site Web : www.ersnet.org/ers/default.aspx?id=3924

\section{ACCÈS AU JOURNAL ÉLECTRONIQUE}

Pour obtenir des directives afin d'accéder au Canadian Respiratory Journal par voie électronique, consultez la page 432.

\section{NOUS AVONS DÉMÉNAGÉ!}

Notre nouvelle adresse est :

Société canadienne de thoracologie

1750, Courtwood Crescent, bureau 300

Ottawa (Ontario)

K2C 2B5

\section{VOUS DÉMÉNAGEZ ? VEUILLEZ NOUS EN AVISER :}

Société canadienne de thoracologie

1750, Courtwood Crescent, bureau 300, Ottawa (Ontario) K1R 1A3

Téléphone : (613) 569-6411, poste 229

Télécopieur : (613) 569-8860, courriel : sdesmarais@lung.ca
The Canadian COPD Alliance

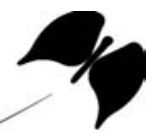
L'Alliance canadienne surla MPOC

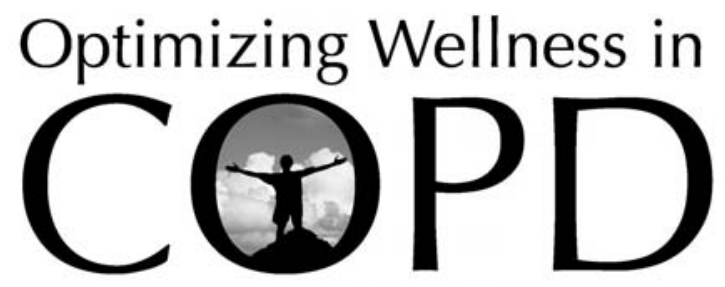




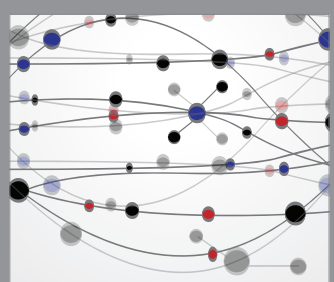

The Scientific World Journal
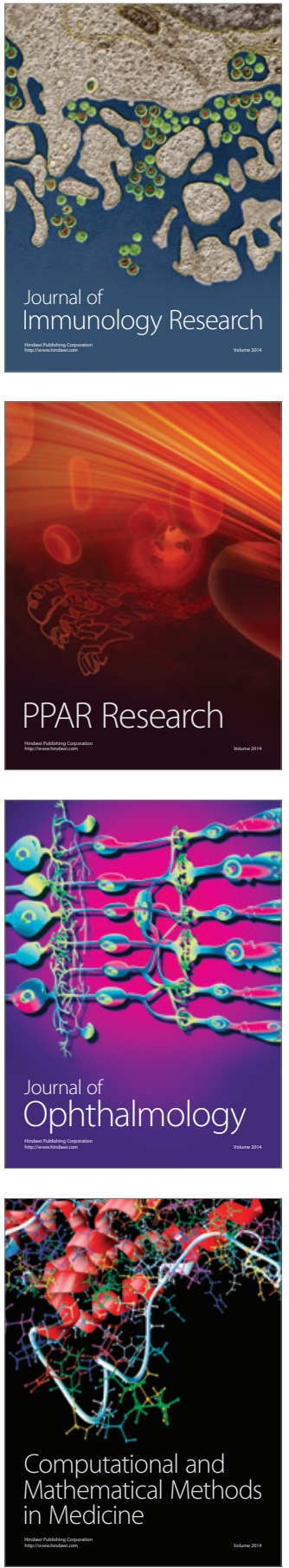

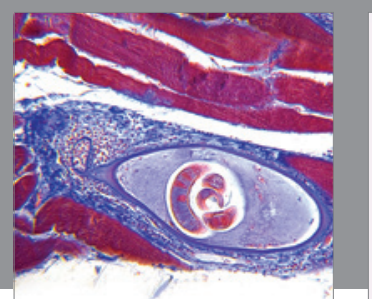

Gastroenterology Research and Practice

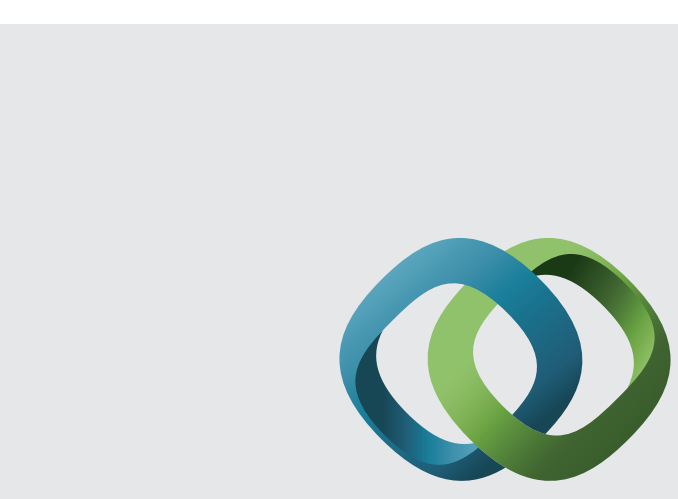

\section{Hindawi}

Submit your manuscripts at

http://www.hindawi.com
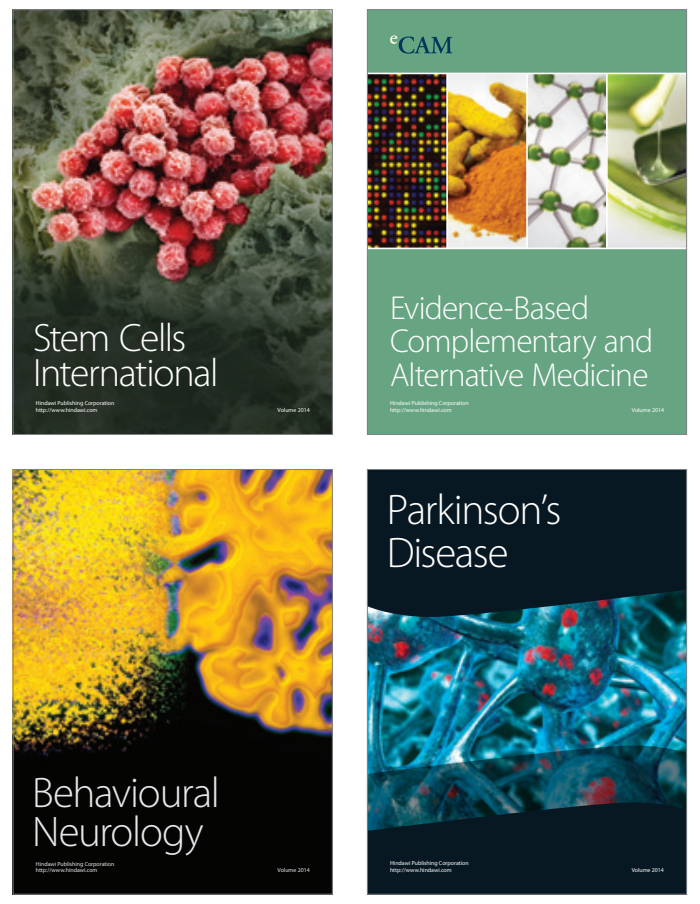
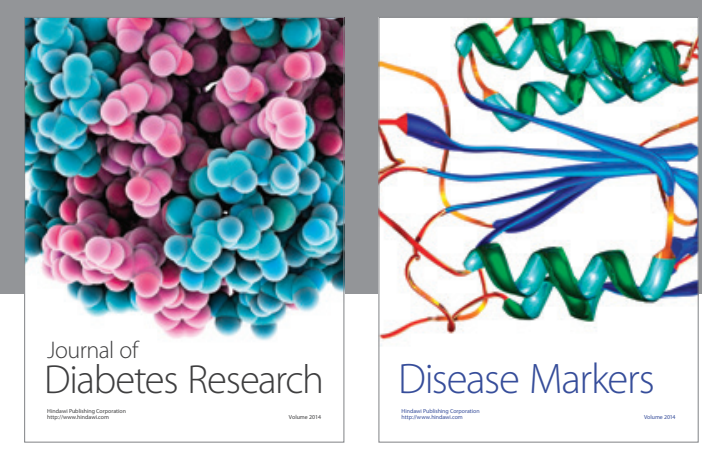

Disease Markers
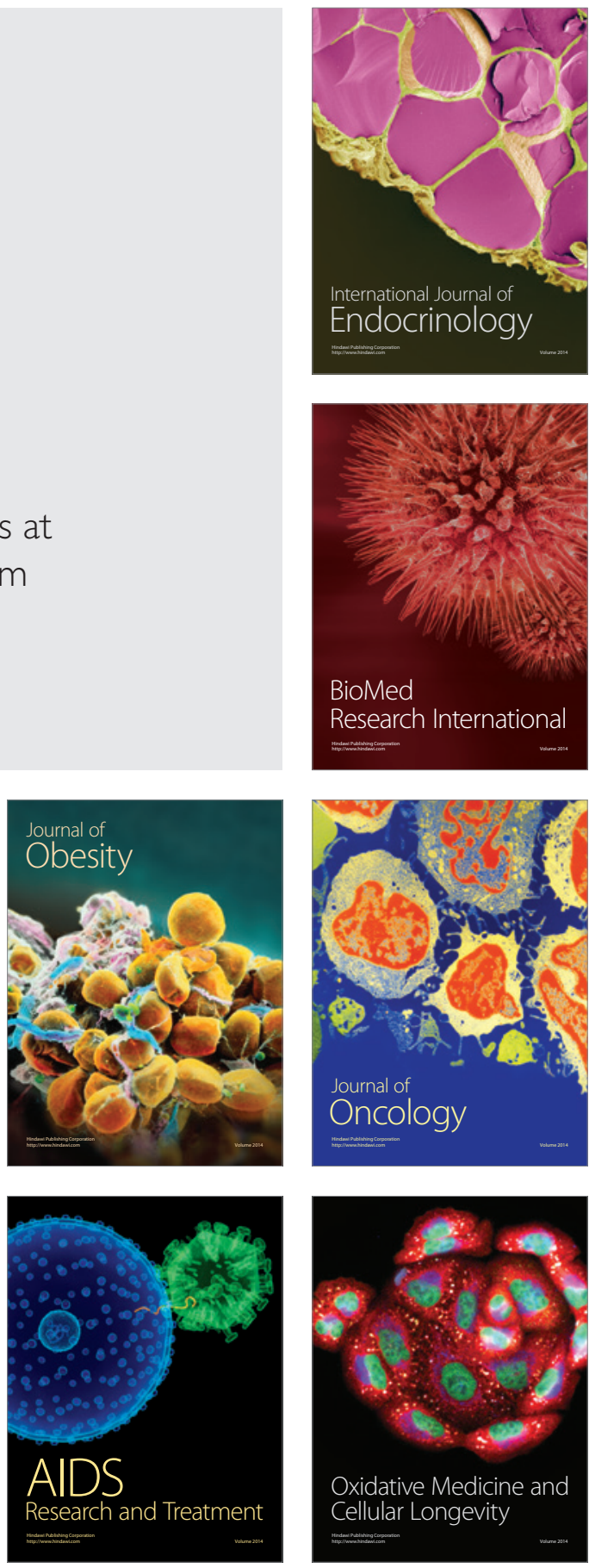